Tohoku J. Exp. Med., 2008, 216, 157-164

\title{
Physician and Hospital Characteristics Related to Length of Stay for Sudden Sensorineural Hearing Loss Patients
}

\author{
Chuan-Song Wu, ${ }^{1}$ Pin-Zhir Chao ${ }^{2}$ and Herng-Ching Lin ${ }^{3}$ \\ ${ }^{1}$ Department of Otolaryngology, Taipei Municipal Chung Hsin Hospital, Taipei, Taiwan \\ ${ }^{2}$ Department of Otolaryngology, Taipei Medical University Hospital, Taipei, Taiwan \\ ${ }^{3}$ School of Health Care Administration, Taipei Medical University, Taipei, Taiwan
}

\begin{abstract}
The treatment of sudden sensorineural hearing loss (SSNHL) has remained one of the most controversial issues in otolaryngology, and there are wide disparities between clinicians' treatment methods for SSNHL. Using five-year population-based data, we explored the relationship between physician and hospital characteristics and the length of stay (LOS) for SSNHL patients in Taiwan. The dataset was derived from the National Health Insurance Research Database. The patients in the study sample were identified by a principal diagnosis of sudden hearing loss. A total of 8712 hospitalization cases between 1998 and 2002 inclusive were included. Multiple regression analyses were performed in order to explore the relationship between physician and hospital characteristics and LOS, adjusting for patients' age and gender, as well as complications or comorbidities. The mean LOS for the duration of the study period was 5.62 days, while the regression analysis showed that, as compared to district hospitals, the LOS in medical centers and regional hospitals was 0.42 and 0.37 days longer, respectively (all $p<0.001$ ). The analysis also revealed that the LOS was 0.71 days shorter for patients who were attended by physicians specializing in otolaryngology than for those attended by physicians specializing in other areas $(p<0.001)$. This study concludes that there is indeed a relationship between physician and hospital characteristics and the LOS for SSNHL patients. The results of this study serve to highlight the importance of developing treatment protocols for SSNHL so as to reduce the variation in physician or hospital behavior. —— length of stay; sudden sensorineural hearing loss; hospital characteristics.
\end{abstract}

Tohoku J. Exp. Med., 2008, 216 (2), 157-164.

(C) 2008 Tohoku University Medical Press

Sudden sensorineural hearing loss (SSNHL) is a well-recognized condition that has perplexed clinicians for a number of decades. Due to insufficient knowledge of the pathophysiology of
SSNHL (Eisenman and Arts 2000), a high spontaneous recovery rate (40 to 65\%) (Mattox and Simmons 1977; Byl 1984), a low incidence (5 to 20 per 100,000) (Fetterman et al. 1996) and the

Received May 8, 2008; revision accepted for publication September 1, 2008.

Correspondence: Herng-Ching Lin, Taipei Medical University, School of Health Care Administration, 250

Wu-Hsing St., Taipei 110, Taiwan.

e-mail: henry11111@tmu.edu.tw

Sources of the study: Secondary data released from Taiwan's National Health Insurance Research Database for the purpose of this study, without patient, institution, or physician identifiers. The study was not funded from any source. 
lack of a universally accepted standard definition for SSNHL (Zadeh et al. 2003), it is difficult to validate empirical treatment and develop an effective treatment protocol. Thus, the treatment of SSNHL has remained one of the most controversial issues in otolaryngology. Furthermore, there are wide disparities between clinicians' treatment methods for SSNHL (Loughran 2000), with treatment being largely dependent on the clinician's personal preference. Clearly, therefore, it is important for otolaryngologists and policymakers alike to determine ways of optimizing SSNHL inpatient treatment, in order to enhance efficient delivery of cost-effective care.

In a cost-conscious healthcare environment, the first stage in optimizing inpatient treatment is gaining an understanding of the factors contributing to the variations in length of stay (LOS), with LOS as the outcome of a complex interaction between patients, physicians and hospitals. An exploration of the sources of variation in LOS will not only help policymakers reduce these differences in order to improve both the efficiency and quality of care, but will also help healthcare professionals define an optimal regimen for treatment. In particular, the management of SSNHL patients using steroids, vasodilators, antiviral agents, diuretics, and low-salt diets varies considerably due to the idiopathic nature of its etiology, so the LOS and quality of care that patients receive might differ greatly among hospitals. However, to our knowledge, no study has yet attempted to investigate the effect of physicians or hospitals on the LOS for SSNHL patients, a state of affairs which clearly prevents otolaryngologists from developing a rational treatment protocol for the condition. Therefore, using a five-year population-based dataset, this study sets out to explore the relationships between physician and hospital characteristics and the LOS for SSNHL patients in Taiwan. In addition to its contribution to the literature in this particular area, our study should also prove useful for future cross-country comparisons.

\section{Methods}

\section{Data Sources}

The dataset for the years 1998 to 2002 comes from the National Health Insurance Research Database (NHIRD), which covers all inpatient and outpatient medical benefit claims for Taiwan's population of over 23 million people. This national database provides us a unique opportunity to examine the effects of physician and hospital characteristics on LOS for SSNHL patients. Taiwan initiated its National Health Insurance (NHI) program in March 1995 to provide healthcare for all of its citizens. The NHI system in Taiwan has a unique combination of characteristics which include universal coverage, a single-payer system (with the government as the sole insurer), and comprehensive benefits coverage; the NHI data is therefore more complete and can better reflect the actual scenario of variations in LOS for SSNHL patients as compared to data from countries which lack universal medical care coverage.

The NHIRD includes registers of contracted medical facilities and board-certified physicians, as well as monthly claims summaries for inpatient and ambulatory care. The database also provides one principal diagnosis code (3882) from the International Classification of Diseases, Ninth Revision, Clinical Modification (ICD-9$\mathrm{CM}$ ) and up to four secondary discharge diagnoses for each patient; in addition, details on all admissions and discharges are precisely recorded.

\section{Study Sample}

We identified all inpatient admission claims from the NHI Research Database (NHIRD), from January 1998 through December 2008, with an ICD-9-CM diagnosis of sudden hearing loss, unspecified (ICD-9-CM code 3882) as principal diagnosis, totaling 10,622 cases. In Taiwan, the possibility of misdiagnosis of SSNHL is very low because of the strict diagnostic criteria for members of the American Academy of OtolaryngologyHead and Neck Surgery, that is, "an acute onset of sensorineural hearing loss at least $30 \mathrm{db}$ in 3 contiguous frequencies within the previous 72 hours (Whitaker 1980)." We also excluded readmissions $(n=1,910)$ since SSNHL often involves more than one admission episode. After excluding the above, our study sample consisted of 8,712 SSNHL patients in Taiwan during 1998-2002.

\section{Key Variables}

The dependent variable of interest in this study was hospital LOS, in days, from admission to discharge. The 
independent variables relating to physicians included gender, age and whether or not the physician specialized in otolaryngology, and variables relating to hospitals included ownership (public, private not-for-profit and private for-profit hospitals), geographic location (north, central, south and east), level (medical centers providing $\geq 500$ beds, regional hospitals with 250-499 beds, district hospitals with 20-249 beds), and teaching status.

Patient variables, which include gender, age and complications or comorbidities, were also controlled for in this study. The sampled patients were categorized into one of three age groups: 0-40, 41-60, and $\geq 61$ years. With regard to complications or comorbidities, all cases were assigned to one of the following five categories: (i) coexisting vertigo or tinnitus; (ii) coexisting diabetes or cardiovascular conditions; (iii) both conditions (i) and (ii) combined; (iv) incidental conditions, pathophysiologically unrelated to SSNHL; and (v) no comorbidity.

\section{Statistical Methods}

All the statistical analyses were performed using the Statistical Package for the Social Sciences (SPSS 10.0 for Windows, 1997, SPSS, Chicago, Ill). One-way analysis of variance (ANOVA) and t-tests were used to examine the relationships between physician and hospital characteristics, and the LOS of SSNHL patients. A multiple regression analysis was also carried out in order to explore the relationship between physician and hospital characteristics and LOS, after adjusting for patient gender, age and complications or comorbidities. A significance level of $p<0.05$ was used in this study.

\section{Results}

The characteristics of physicians, hospitals and SSNHL patients sampled in this study are summarized in Table 1, which shows that the LOS for SSNHL patients ranged from 1 to 43 days, with a mean of 5.62 days and a standard deviation of 2.74 days. Medical centers (representing $52.7 \%$ of the sample) had the highest share of SSNHL hospitalization, while district hospitals $(8.3 \%)$ had the lowest share. The mean age of physicians was $39.33( \pm 7.34)$ years, with an age range of 29 to 68 years. Among the attending physicians, $87.5 \%$ were otolaryngologists, while the 'others' included physicians specializing in internal medicine, neurology, family practice and general practice. Patients' ages ranged from 3 to
91 years, with a mean age of 49.3 years and a standard deviation of 16.8 years.

Details of the unadjusted LOS for patients, by hospital and physician characteristics, are provided in Table 2. ANOVA showed that the LOS for patients hospitalized as a result of SSNHL was significantly correlated to hospital level $(p<0.01)$, hospital ownership $(p<0.001)$ and hospital location $(p<0.001)$. In addition, the t-test demonstrated a significant association between hospital teaching status $(p<0.01)$, physician specialty $(p<0.01)$ and SSNHL LOS. The mean LOS for patients of physicians specializing in otolaryngology was 5.58 days, while the mean stay for patients of 'other' physicians was 5.88 days.

Table 3 presents the details of the adjusted relationship between hospital and physician characteristics and the LOS for SSNHL patients. Since all medical centers and regional hospitals are, by nature, teaching hospitals, teaching status was not included in this regression. After adjusting for patient gender, age and complications or comorbidities, analysis revealed that the LOS for both medical centers and regional hospitals was longer and more statistically significant than the LOS for district hospitals. It also revealed that the LOS tended, on average, to be significantly longer for SSNHL patients hospitalized in southern Taiwan than for those hospitalized in other parts of Taiwan.

Table 3 also shows that the LOS among sampled patients attended by physicians specializing in otolaryngology was more likely to be shorter, on average, than for patients attended by physicians specializing in other areas. As expected, the LOS for patients suffering SSNHL with coexisting vertigo, tinnitus, diabetes or cardiovascular conditions, was longer than that for other patients. It is also worth noting that male patients suffering from SSNHL had significantly longer LOS than their female counterparts.

\section{Discussion}

SSNHL is one of the most controversial and, as yet, unsolved issues in otolaryngology, largely due to the insufficient number of populationbased empirical studies, the limited understanding 
TABLE 1. Descriptive statistics of sudden sensorineural hearing loss hospitalization from 1998 to 2002 in Taiwan $(n=8,712)$

\begin{tabular}{|c|c|c|}
\hline Variables & $n$ & $\%$ \\
\hline \multicolumn{3}{|l|}{ Hospital level } \\
\hline Medical center & 4,595 & 52.7 \\
\hline Regional hospital & 3,396 & 39.0 \\
\hline District hospital & 721 & 8.3 \\
\hline \multicolumn{3}{|l|}{ Hospital ownership } \\
\hline Public & 3,957 & 45.4 \\
\hline NFP & 3,666 & 42.1 \\
\hline FP & 1,089 & 12.5 \\
\hline \multicolumn{3}{|l|}{ Hospital location } \\
\hline North & 4,380 & 50.3 \\
\hline Central & 2,345 & 26.9 \\
\hline South & 1,838 & 21.1 \\
\hline East & 149 & 1.7 \\
\hline \multicolumn{3}{|l|}{ Teaching status } \\
\hline Yes & 8,373 & 96.1 \\
\hline No & 339 & 3.9 \\
\hline \multicolumn{3}{|l|}{ Patient's age (year) } \\
\hline $0-40$ & 2,579 & 29.6 \\
\hline $41-60$ & 3,658 & 42.0 \\
\hline$\geq 61$ & 2,475 & 28.4 \\
\hline \multicolumn{3}{|l|}{ Patient's gender } \\
\hline Male & 4,734 & 54.3 \\
\hline Female & 3,978 & 45.7 \\
\hline \multicolumn{3}{|l|}{ Complications or comorbidities } \\
\hline Vertigo or tinnitus & 380 & 4.4 \\
\hline Diabetes or Cardiovascular diseases & 1,201 & 13.8 \\
\hline Vertigo/tinnitus and Diabetes/Cardiovascular diseases & 69 & 0.8 \\
\hline Other complications & 1,585 & 18.2 \\
\hline No complication & 5,477 & 62.9 \\
\hline \multicolumn{3}{|l|}{ Physician gender } \\
\hline Male & 8,355 & 95.9 \\
\hline Female & 357 & 4.1 \\
\hline \multicolumn{3}{|l|}{ Physician specialty } \\
\hline Otolaryngology & 7,620 & 87.5 \\
\hline Others & 1,092 & 12.5 \\
\hline
\end{tabular}

LOS, length of stay; FP, for profit; NFP, not for profit; s.D., standard deviation. 
TABLE 2. Analysis of length of stay by hospital and physician characteristics $(n=8,712)$

\begin{tabular}{|c|c|c|c|c|c|c|}
\hline \multirow{2}{*}{ Variables } & \multicolumn{6}{|c|}{ Length of stay } \\
\hline & $n$ & Mean & S.D. & Min & $\operatorname{Max}$ & $\mathrm{t}(\mathrm{F})$-test \\
\hline Hospital level & & & & & & $5.54 * *$ \\
\hline Medical center & 4,595 & 5.72 & 2.23 & 1 & 43 & \\
\hline Regional hospital & 3,396 & 5.64 & 2.54 & 1 & 40 & \\
\hline District hospital & 722 & 5.42 & 2.38 & 1 & 29 & \\
\hline Hospital ownership & & & & & & $34.35 * * *$ \\
\hline Public & 3,957 & 5.84 & 2.64 & 1 & 40 & \\
\hline NFP & 3,666 & 5.48 & 2.16 & 1 & 43 & \\
\hline FP & 1,089 & 5.31 & 1.91 & 1 & 16 & \\
\hline Hospital location & & & & & & $47.65 * * *$ \\
\hline North & 4,380 & 5.67 & 2.46 & 1 & 43 & \\
\hline Central & 2,345 & 5.20 & 2.14 & 1 & 26 & \\
\hline South & 1,838 & 6.06 & 2.36 & 1 & 28 & \\
\hline East & 149 & 5.30 & 2.39 & 1 & 14 & \\
\hline Teaching status & & & & & & $-2.21 * *$ \\
\hline Yes & 8,373 & 5.72 & 2.78 & 1 & 43 & \\
\hline No & 339 & 5.48 & 1.71 & 1 & 14 & \\
\hline Physician gender & & & & & & -1.28 \\
\hline Male & 8,355 & 5.63 & 2.37 & 1 & 43 & \\
\hline Female & 357 & 5.46 & 2.36 & 1 & 27 & \\
\hline Physician specialty & & & & & & $5.34 * *$ \\
\hline Otolaryngology & 7,620 & 5.58 & 2.25 & 1 & 43 & \\
\hline Others & 1,092 & 5.88 & 3.11 & 1 & 40 & \\
\hline
\end{tabular}

NFP, not-for-profit; FP, for-profit; S.D., standard deviation; $* * p<0.01$; ***p $<0.001$.

of the etiology of SSNHL, and the absence of any universally agreed upon treatment protocol. We have found that, after adjusting for patient gender, age and complications or comorbidities, a significant relationship does exist between the LOS for SSNHL patients and specific physician and hospital characteristics. Our findings should be understood in light of the conclusions of a UK survey of one hundred otolaryngologists by Loughran (2000), which found wide variations between practitioners with regard to the methods and treatment combinations used for the overall treatment of SSNHL.

We have found that a substantial majority of patients suffering from SSNHL (51.3\%) were hospitalized in medical centers, which, in Taiwan, are generally considered as places for treating ill- nesses of greater severity. Unlike the health plans under the managed care system in the US, which place strict limits on patients' choice of providers, patients in Taiwan are free to go to any medical institution of their choice. Therefore, it would seem that the high proportion of admissions of patients suffering from SSNHL to medical centers suggests patients in Taiwan regard SSNHL as a serious condition. This is not, however, too surprising, since SSNHL is an abrupt or rapidly progressive sensorineural hearing impairment, and such rapid onset of the condition will clearly be a frightening experience, causing considerable anxiety for patients affected by the condition.

This study has found that patients admitted to medical centers and regional hospitals generally had longer LOS than patients admitted to dis- 
TABLE 3. Adjusted relationship between length of stay and hospital and physician characteristics $(n=8,712)$

\begin{tabular}{|c|c|c|c|}
\hline Variables & B & SE & t-test \\
\hline \multicolumn{4}{|l|}{ Hospital level } \\
\hline Medical center & 0.42 & 0.08 & $5.25^{* * *}$ \\
\hline Regional hospital & 0.37 & 0.08 & $4.75^{* * *}$ \\
\hline \multicolumn{4}{|l|}{ District hospital (reference group) } \\
\hline \multicolumn{4}{|l|}{ Hospital ownership } \\
\hline Public & 0.13 & 0.10 & 1.29 \\
\hline NFP & 0.06 & 0.10 & 0.62 \\
\hline \multicolumn{4}{|l|}{ FP (reference group) } \\
\hline \multicolumn{4}{|l|}{ Hospital location } \\
\hline \multicolumn{4}{|l|}{ North (reference group) } \\
\hline Central & -0.36 & 0.07 & $-5.13 * * *$ \\
\hline South & 0.46 & 0.07 & $6.80^{* * *}$ \\
\hline East & -0.22 & 0.19 & -1.21 \\
\hline \multicolumn{4}{|l|}{ Patient's age } \\
\hline $0-40$ & -0.04 & 0.06 & -0.63 \\
\hline \multicolumn{4}{|l|}{ 41-60 (reference group) } \\
\hline$\geq 65$ & 0.01 & 0.06 & -0.22 \\
\hline \multicolumn{4}{|l|}{ Patient's gender } \\
\hline Male & 0.18 & 0.05 & $3.58 * * *$ \\
\hline \multicolumn{4}{|l|}{ Female (reference group) } \\
\hline Physician's age & 0.02 & 0.00 & $5.44 * * *$ \\
\hline \multicolumn{4}{|l|}{ Physician's specialty } \\
\hline Otolaryngology & -0.71 & 0.07 & $-10.14 * * *$ \\
\hline \multicolumn{4}{|l|}{ Others (reference group) } \\
\hline \multicolumn{4}{|l|}{ Complication or comorbidities } \\
\hline Vertigo or tinnitus & 0.25 & 0.13 & $2.03 *$ \\
\hline Diabetes or Cardiovascular diseases & 0.57 & 0.08 & $7.31 * * *$ \\
\hline Vertigo/tinnitus and Diabetes/Cardiovascular Diseases & 1.25 & 0.28 & $4.40 * * *$ \\
\hline Other complications & 0.52 & 0.07 & $7.80^{* * *}$ \\
\hline \multicolumn{4}{|l|}{ No complication (reference group) } \\
\hline Constant & 4.46 & 0.22 & $20.49 * * *$ \\
\hline
\end{tabular}

NFP, not-for-profit; FP, for-profit; s.D., standard deviation; ${ }^{* *} p<0.01$; ${ }^{* * *} p<0.001$.

trict hospitals. More specifically, the adjusted LOS for both medical centers and regional hospitals was about $11 \%(0.52 / 4.46)$ higher than for district hospitals. This phenomenon can be explained partly by the tendency of medical centers and regional hospitals to receive a relatively higher proportion of patients suffering from serious illnesses than district hospitals. Although advanced adjustments were made in this study for complications or comorbidities, there was no plausible method for ensuring that all sampled patients had the same unmeasured severity of illness. Our interpretation, however, is supported by Samuels et al.'s study which indicated that 'adverse case mix/severity of illness' was a major contributory factor to the variations in LOS between hospitals (Samuels et al. 1998). The study by Yimtae et al. (2001) also demonstrated 
that the severity of hearing loss significantly influenced the prognosis for patients suffering from SSNHL.

Rather interestingly, we find that the LOS for SSNHL patients attended by otolaryngologists was $18.2 \%$ shorter than the LOS for those attended by physicians with other specialties. A possible explanation for this is that otolaryngologists are more likely than other physicians to admit and treat such patients; a high volume of patients will provide otolaryngologists with more practical experience in the effective and efficient treatment of SSNHL. However, it is also possible that patients cared for by otolaryngologists would tend to be more severely ill than those cared for by physicians with other specialties. A well-designed questionnaire survey is thus required to determine whether there are differences in understanding, attitudes or treatment methods for SSNHL between otolaryngologists and other physicians.

The analysis also revealed that the LOS for patients hospitalized as a result of SSNHL in southern Taiwan tended, on average, to be significantly longer than those in northern Taiwan. It could be that there is a high density of hospitals concentrated in northern Taiwan compared to southern Taiwan, and the competitive pressures may encourage hospitals in northern Taiwan to accommodate patient preferences for longer LOSs.

Not surprisingly, this study has revealed that patients with comorbidities of vertigo or tinnitus had, on average, an LOS which was 0.79 days $(17.7 \%)$ longer than that of other patients. This is comparable to the findings of Uri et al. (2003) and Byl (1984), who found vertigo or tinnitus to be an important factor relating to poor prognosis. A possible reason for the longer LOS for SSNHL patients with complications of vertigo or tinnitus could be more extensive disease of the inner ear. Similarly, we also found that a coexisting condition of diabetes was contributory factor to the longer LOS for SSNHL patients. This finding comes in light of a previous study by Shikowitz (1991), which found that diabetes was an important factor in the prognosis of SSNHL patients.

We should make note of one major limitation inherent within this study. First of all, while the prior studies have reported that the level of severity of hearing loss, the interval between onset and treatment, and the audiogram type, were all important predictors of recovery for patients suffering from SSNHL (Eisenman and Arts 2000; Moskowitz et al. 1984), unfortunately, this information is not available from the NHIRD. Further research should therefore focus on collecting more detailed information regarding hospital admission policies, patients' disease characteristics, and physicians' behavior in order to make more specific interpretations of the variation in LOS observed in the present study.

\section{Conclusions}

This study indicates that a significant relationship does indeed exist between physician and hospital-related characteristics and the LOS for SSNHL patients, after adjusting for patients' gender, age and complications or comorbidities. This study has also demonstrated, however, wide variations between physicians and hospitals in the LOS for SSNHL patients. Although it is almost impossible to determine a most 'appropriate' LOS for SSNHL hospitalization, the results of this study should highlight the importance of developing treatment protocols or practice guidelines for SSNHL so as to reduce variations in hospital or physician behavior.

\section{Acknowledgments}

This study is based in part on data from the National Health Insurance Research Database provided by the Bureau of National Health Insurance, Department of Health, Taiwan and managed by the National Health Research Institutes. The interpretations and conclusions contained herein do not represent those of the Bureau of National Health Insurance, Department of Health, or the National Health Research Institutes.

\section{References}

Byl, F.M. Jr. (1984) Sudden hearing loss: eight years' experience and suggested prognostic table. Laryngoscope, 94, 647-661.

Eisenman, D. \& Arts, H.A. (2000) Effectiveness of treatment for sudden sensorineural hearing loss. Arch. Otolaryngol. Head Neck Surg., 126, 1161-1164.

Fetterman, B.L., Luxford, W.M. \& Saunders, J.E. (1996) 
Sudden bilateral sensorineural hearing loss. Laryngoscope, 106, 1347-1350.

Loughran, S. (2000) Management of sudden sensorineural hearing loss: a consultant survey. J. Laryngol. Otol., 114, 837-839.

Mattox, D.E. \& Simmons, F.B. (1977) Natural history of sudden sensorineural hearing loss. Ann. Otol. Rhinol. Laryngol., 86, 463-480.

Moskowitz, D., Lee, K.J. \& Smith, H.W. (1984) Steroid use in idopathic sudden sensorineural hearing loss. Laryngoscope, 94, 664-666.

Samuels, B.N., Novack, A.H., Martin, D.P. \& Connell, F.A. (1998) Comparison of length of stay for asthma by hospital type. Pediatrics., 101, E13.

Shikowitz, M.J. (1991) Sudden sensorineural hearing loss.
Med. Clin. North. Am., 75, 1239-1250.

Uri, N., Doweck, I., Cohen-Kerem, R. \& Greenberg, E. (2003) Acyclovir in the treatment of idiopathic sudden sensorineural hearing loss. Otolaryngol. Head Neck Surg., 128, 544-549.

Whitaker, S. (1980) Idiopathic sudden hearing loss. Am. J. Otol., 1, 180-183.

Yimtae, K., Srirompotong, S. \& Kraitrakul, S. (2001) Idiopathic sudden sensorineural hearing loss. J. Med. Assoc. Thai., 84, 113-119.

Zadeh, M.H., Storper, I.S. \& Spitzer, J.B. (2003) Diagnosis and treatment of sudden-onset sensorineural hearing loss: a study of 51 patients. Otolaryngol. Head Neck Surg., 28, 92-98. 